\title{
Hyperphosphatemia, CTCAE
}

National Cancer Institute

\section{Source}

National Cancer Institute. Hyperphosphatemia, CTCAE. NCI Thesaurus. Code C143222.

A disorder characterized by laboratory test results that indicate an elevation in the concentration of phosphate in a blood. 